\title{
A atuação da Atenção Primária no contexto da pandemia da COVID-19 no Brasil
}

\author{
The role of Primary Care in the context of the COVID-19 pandemic in Brazil \\ El papel de la Atención Primaria en el contexto de la pandemia COVID-19 en Brasil
}

Recebido: 19/06/2021 | Revisado: 29/06/2021 | Aceito: 04/07/2021 | Publicado: 15/07/2021

\begin{abstract}
Resumo
O presente estudo realizou uma revisão integrativa de literatura sobre a atuação da atenção primária à saúde no Brasil no contexto da pandemia da Covid-19. A coleta de dados foi realizada nas bases de dados: LILACS, SCIELO e portal da CAPES. Os descritores utilizados foram: "Atenção Primária à Saúde", "Estratégia Saúde da Família", "Covid -19" associados entre si pelo operador booleano AND. Foram incluídos os artigos relacionados ao tema do estudo, disponíveis na integra, no idioma português e publicados no período de janeiro de 2020 a março de 2021. Foram excluídos os protocolos ou documentos técnicos, teses, dissertações e monografias. Os artigos incluídos na amostra foram lidos na íntegra para identificação, descrição e análise dos temas abordados. Observou-se que as estratégias de enfrentamento à Covid-19 na atenção primária à saúde do Brasil permearam dois aspectos: a reorganização no processo de trabalho e as ações e atividades desenvolvidas, enfatizando a educação em saúde por produzir conhecimentos oportunizando o autocuidado. Os resultados desse estudo evidenciaram o potencial da atenção primária à saúde no enfretamento à Covid-19 ressaltando a importância do seu fortalecimento, especialmente nesse período de pandemia.
\end{abstract}

Palavras-chave: Atenção primária em saúde; Estratégia saúde da família; Covid-19.

\begin{abstract}
This study carried out an integrative literature review on the role of primary health care in Brazil in the context of the Covid-19 pandemic. Data collection was performed in the databases: LILACS, SCIELO and CAPES portal. The descriptors used were: "Primary Health Care", "Family Health Strategy", "Covid-19" associated with each other by the Boolean operator AND. Articles related to the topic of the study, available in full, in Portuguese and published from january 2020 to march 2021, were included. Protocols or technical documents, theses, dissertations and monographs were excluded. The articles included in the sample were read in full for identification, description and analysis of the topics covered. It was observed that coping strategies against Covid-19 in primary health care in Brazil permeated two aspects: the reorganization of the work process and the actions and activities developed, emphasizing health education for producing knowledge and providing opportunities for self-care. The results of this study highlighted the potential of primary health care in confronting Covid-19, highlighting the importance of strengthening it, especially in this period of pandemic.
\end{abstract}

Keywords: Primary health care; Family health strategy; Covid-19.

\section{Resumen}

Este estudio realizó una revisión integradora de la literatura sobre el papel de la atención primaria de salud en Brasil en el contexto de la pandemia de Covid-19. La recolección de datos se realizó en las bases de datos, portal LILACS, SCIELO y CAPES. Los descriptores utilizados fueron: "Atención Primaria de Salud”, "Estrategia de Salud de la Familia", "Covid-19" asociados entre sí por el operador booleano AND. Se incluyeron artículos relacionados con el tema del estudio, disponibles íntegramente, en portugués y publicados desde enero de 2020 hasta marzo de 2021 . Se excluyeron los protocolos o documentos técnicos, tesis, disertaciones y monografías. Los artículos incluidos en la muestra fueron leídos íntegramente para la identificación, descripción y análisis de los temas tratados. Se observó que las estrategias de afrontamiento al Covid-19 en la atención primaria de salud en Brasil permearon dos aspectos: la reorganización del proceso de trabajo y las acciones y actividades desarrolladas, enfatizando la educación para la salud para producir conocimiento y brindar oportunidades de autocuidado. Los resultados de este estudio destacaron 
el potencial de la atención primaria de salud para enfrentar el Covid-19, destacando la importancia de fortalecerlo, especialmente en este período de pandemia.

Palabras clave: Atención primaria de salud; Estrategia de salud familiar; Covid-19.

\section{Introdução}

Em dezembro de 2019, o surgimento de uma doença respiratória mobilizou as autoridades sanitária de Wuhan, cidade situada na província de Hubei, na China. Tratava-se de uma infecção causada por um novo tipo de coronavírus, que mais tarde, foi nomeado como SARS-CoV-2 (Severe Acute Respiratory Syndrome 2), o agente etiológico da doença Covid-19 (Coronavírus Disease) (They, 2020). A Covid-19 é caracterizada por uma síndrome respiratória com espectro clínico variando de casos assintomáticos à graves (Brasil, 2020).

No Brasil, o primeiro caso de Covid-19 foi registrado em 25 de fevereiro de 2020 e o primeiro óbito em 17 de março do mesmo ano. No dia 20 de março de 2020, o Ministério da Saúde declarou transmissão comunitária em todo território nacional (They, 2020). A Covid-19 se propagou incialmente nas grandes metrópoles brasileiras e seguiu para o interior, por se tratar de um país com dimensão continental e realidades sociais discrepantes (Amaral et al, 2020).

Os pacientes com Covid-19 em estado grave podem necessitar de unidade de terapia intensiva (UTI) e considerando que a disponibilidade de vagas em leitos de UTI é escassa em algumas localidades tendo em vista as desigualdades regionais do país, é iminente o risco de óbito por falta de uma assistência adequada (Noronha et al, 2020). Embora apresente uma baixa taxa de letalidade, cerca de 0,6\%, dos casos confirmados de Covid-19, segundo a OMS, a Covid-19 preocupa as autoridades pela rapidez com que o vírus se propaga atingindo facilmente uma grande parcela da população, que adoecendo ao mesmo tempo pode causar um colapso no sistema de saúde (They, 2020).

Entretanto, segundo a OMS cerca de 80\% dos casos de Covid-19 são considerados leves ou moderados, ou seja, não necessitam de internação hospitalar, podendo ser acompanhados, pela Atenção Primária à Saúde (APS). Nesse sentido, uma APS forte tem capacidade de contribuir de modo importante no enfrentamento de situações de emergências públicas e isso pode ser evidenciado na eficiência da APS no cuidado em saúde da população, que vem apresentando resultados bastante satisfatórios no enfrentamento às arboviroses, no combate à mortalidade materna e infantil, no controle das doenças crônicas, entre outras, decorrente de sua capilaridade e do conhecimento do território, que fortalece o vínculo entre a equipe de saúde e a comunidade, contribuindo para integralidade da assistência (Sarti et al, 2020).

Segundo Alves (2020), a Atenção Primária no Sistema Único de Saúde (SUS) é o nível de atenção mais capilarizado e através da Estratégia Saúde da Família (ESF), alcança a maioria dos municípios brasileiros, possibilitando transversalmente efetiva intervenção no controle da Covid-19, por seu alto potencial de resolubilidade nos problemas de saúde da população, sendo preferencialmente o primeiro contato do usuário com o serviço de saúde acrescido ao vínculo longitudinal que auxilia na tomada de decisão. Sendo assim, é importante ressaltar que um dos objetivos da APS é a diminuição das internações hospitalares e a ESF vem produzindo saúde de forma singular, através do diagnóstico de seu território e da sua população adscrita, garantindo-lhes acesso à serviços e ações de promoção, prevenção e recuperação da saúde. Esse arranjo organizativo alcança os diferentes grupos populacionais de modo peculiar, tal qual, nenhum dispositivo foi tão eficiente até então (Barbosa, 2020).

Considerando as diretrizes da atenção primária à saúde, tais como: Regionalização, Hierarquização, Territorialização, População Adscrita, Cuidado Centrado na pessoa, Resolutividade, Longitudinalidade do Cuidado, Ordenação da Rede e Participação da Comunidade é imprescindível considerá-la um importante dispositivo de enfrentamento à Covid-19 (Brasil, 2017). Entretanto, vale salientar que a APS tem enfrentado alguns retrocessos nos últimos anos, especialmente com a modificação da Política Nacional de Atenção Básica, em 2017. Uma das mudanças mais preocupantes foi a substituição da diretriz "cuidado centrado na família" pelo "cuidado centrado na pessoa”, direcionando o foco para o indivíduo e a clínica, não 
valorizando a dimensão comunitária da APS que é imprescindível e estratégica no enfrentamento da pandemia (Melo et al, 2018). Além disso, a não obrigatoriedade da presença do agente comunitário de saúde (ACS) e a mudança no financiamento da APS que pode interferir na manutenção de equipes multiprofissionais ampliadas como as do Núcleo Ampliado de Saúde da Família e Atenção Básica (NASF-AB) representam alguns dos retrocessos sofridos pela APS nos últimos anos (Giovanella, Franco \& Almeida, 2020; Morosini, Fonseca \& Baptista, 2020).

Sem perder de vista esse cenário recente de ataques à APS e considerando o seu papel estratégico na efetivação do acesso integral a saúde da população, esse trabalho objetiva analisar a atuação da atenção primária no enfrentamento da pandemia da Covid-19 no Brasil.

\section{Metodologia}

Realizou-se um estudo de revisão integrativa de literatura com abordagem qualitativa. A revisão integrativa é um dispositivo singular no campo da saúde, por sintetizar estudos disponíveis a respeito de determinado tema conduzindo à prática, fundamentado em evidências científicas (Souza et al., 2010).

A pesquisa foi realizada no período de janeiro a abril de 2021 e a coleta de dados foi realizada através de artigos publicados no Scientific Electronic Library Online (SCIELO), Literatura Latino-Americana e do Caribe em Ciências da Saúde (LILACS) e Portal de periódicos da Coordenação de Aperfeiçoamento de Pessoal de Nível Superior (CAPES). Os descritores utilizados foram: “Atenção Primária à Saúde", "Estratégia Saúde da Família”, "Covid-19” associados entre si pelo operador booleano AND.

Após a identificação dos artigos, foram incluídos apenas os que estavam relacionados ao tema do estudo e atenderam os seguintes critérios: i) Disponibilizados na íntegra; ii) Publicados em português; iii) Publicados no período de janeiro de 2020 a março de 2021. Após a identificação dos textos, foram excluídos: i) Protocolos ou documentos técnicos; ii) Tese, dissertações e monografias. A figura 1 demonstra graficamente como se deu o fluxo da coleta de dados desse trabalho onde foram identificados 199 estudos e selecionados 13, sendo grande parte dos textos excluídos por ser fora do tópico de interesse ou se tratar de protocolos. Os artigos incluídos na amostra foram lidos na íntegra para identificação, descrição e análise dos temas abordados que foram classificados em 3 categorias de forma indutiva: Mudança no processo de trabalho; Atividades desenvolvidas e Dificuldades encontradas, permitindo compilar o aprendizado construído sobre o tema. 
Figura 1 - Fluxograma da coleta de dados.

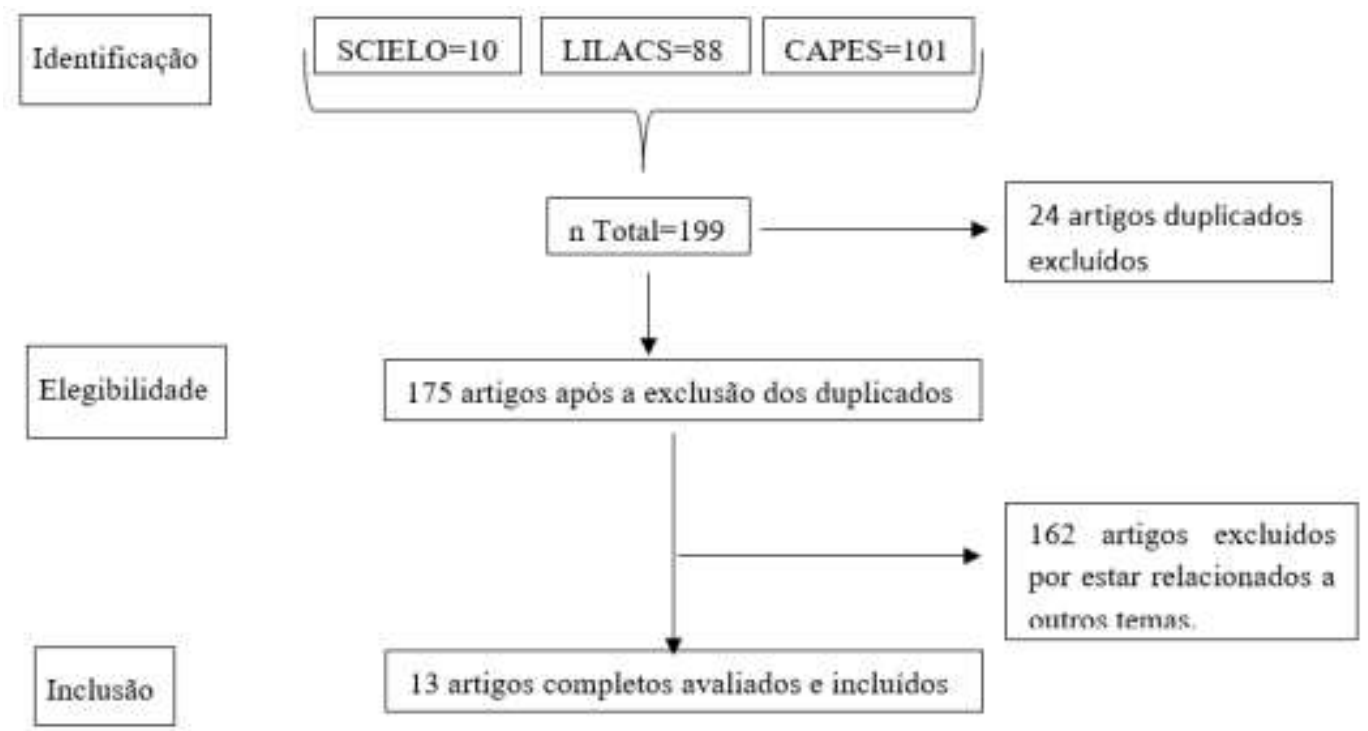

Fonte: Autores (2021).

\section{Resultados e Discussão}

\subsection{Caracterização dos estudos selecionados sobre atenção primária à saúde no enfrentamento da Covid-19 no Brasil}

O presente trabalho selecionou 13 estudos descritivos (Quadro 1). Destes, sete são relatos de experiência (E5, E6, E7, E8, E9, E10, E12) que possibilitam conhecer a realidade local da atuação APS durante a pandemia nos municípios estudados. Os resultados desse tipo de estudo são insuficientes para descrever uma realidade abrangente. Mas, considerando o pouco tempo decorrido entre o início da pandemia no Brasil e a publicação dos primeiros artigos, tornam-se textos importantes para identificar aspectos semelhantes ou singulares do cotidiano das equipes da atenção primária no enfrentamento da Covid-19. Esses relatos de experiência estão distribuídos em diferentes localidades e regiões, sendo 05 estudos da região Nordeste, 01 da região Sul e 01 da região Sudeste. Os demais estudos são descritivos (E1, E2, E3, E4, E11 e E13) e foram realizados no Rio de Janeiro.

Os estudos buscaram refletir acerca da atuação da atenção primária durante a pandemia de forma geral e em algumas atividades mais especificas, como a saúde materno-infantil e a saúde bucal. Um aspecto relevante encontrado nos estudos foi o papel estratégico da APS na atuação direta de enfrentamento a pandemia, confirmando sua missão. Há uma série de descrições da realidade de alguns municípios brasileiros sobre como as equipes se adaptaram às novas demandas, agregaram novos dispositivos, formaram parcerias e uniu saberes integrando-se à vigilância em saúde a fim de potencializar ações de monitoramentos de casos e educação em saúde.

Ademais, é pertinente mencionar o posicionamento de alguns estudos na reafirmação e defesa do SUS no enfrentamento à essa pandemia, tendo em vista todo desmonte que vem sofrendo e na necessidade de tornar esse pleito coletivo. No quadro 1 é possível observar alguns aspectos dos estudos selecionados. 
Quadro 1 - Caracterização dos artigos selecionados.

\begin{tabular}{|c|c|c|c|c|c|}
\hline $\begin{array}{l}\text { Identifi- } \\
\text { cação }\end{array}$ & Título do estudo & $\begin{array}{l}\text { Local/ } \\
\text { Ano }\end{array}$ & Objetivo do estudo & $\begin{array}{l}\text { Tipo do } \\
\text { estudo }\end{array}$ & Principais resultados \\
\hline E1 & $\begin{array}{l}\text { Gestante no contexto da } \\
\text { pandemia da Covid-19: } \\
\text { reflexões e desafios }\end{array}$ & $\begin{array}{l}\text { Rio de } \\
\text { Janeiro } \\
2020\end{array}$ & $\begin{array}{c}\text { Refletir sobre estar gestante em } \\
\text { período de pandemia e a } \\
\text { importância do cuidado } \\
\text { profissional para superar os } \\
\text { desafios que permeiam esse } \\
\text { contexto } \\
\end{array}$ & $\begin{array}{c}\text { Estudo } \\
\text { descritivo }\end{array}$ & $\begin{array}{c}\text { Faz-se necessário planejar novas } \\
\text { estratégias para atender as } \\
\text { demandas das gestantes } \\
\text { considerando as constantes } \\
\text { mudanças na rede de saúde }\end{array}$ \\
\hline E2 & $\begin{array}{l}\text { A atenção primária no } \\
\text { Rio de Janeiro em } \\
\text { tempos de Covid-19 }\end{array}$ & $\begin{array}{l}\text { Rio de } \\
\text { Janeiro } \\
2020\end{array}$ & $\begin{array}{c}\text { Abordar a grave situação da APS } \\
\text { no Rio de Janeiro, destacando } \\
\text { alguns fatores que contribuíram } \\
\text { para esse agravamento }\end{array}$ & $\begin{array}{l}\text { Estudo } \\
\text { descritivo }\end{array}$ & $\begin{array}{l}\text { A pandemia apenas expôs a grave } \\
\text { situação da saúde no Rio de } \\
\text { Janeiro, com agravamento nas } \\
\text { comunidades e periferias }\end{array}$ \\
\hline E3 & $\begin{array}{l}\text { A atenção do dentista da } \\
\text { família na pandemia } \\
\text { Do Covid-19, o cenário } \\
\text { do Rio de Janeiro }\end{array}$ & $\begin{array}{l}\text { Rio de } \\
\text { Janeiro } \\
2020\end{array}$ & $\begin{array}{l}\text { Refletir a respeito da atuação do } \\
\text { dentista de família durante a } \\
\text { pandemia, ponderando a atual } \\
\text { competência técnica com a real } \\
\text { possibilidade de ampliar o escopo } \\
\text { de trabalho }\end{array}$ & $\begin{array}{c}\text { Estudo } \\
\text { descritivo }\end{array}$ & $\begin{array}{l}\text { Existe demanda e respaldo técnico } \\
\text { que justifique a ampliação da } \\
\text { atuação do dentista da família no } \\
\text { combate à Covid-19, fazendo-se } \\
\text { necessário superar paradigmas que } \\
\text { o restringe seu trabalho a } \\
\text { procedimentos clínicos }\end{array}$ \\
\hline E4 & $\begin{array}{l}\text { Sobre o jogo de (des) } \\
\text { montar: Covid-19 e a } \\
\text { atenção primária à } \\
\text { saúde }\end{array}$ & $\begin{array}{l}\text { Rio de } \\
\text { Janeiro } \\
2020\end{array}$ & $\begin{array}{c}\text { Refletir sobre o impacto do } \\
\text { desmonte do SUS suas } \\
\text { consequências sobre a pandemia } \\
\text { e a potência desse modelo público } \\
\text { de saúde }\end{array}$ & $\begin{array}{c}\text { Estudo } \\
\text { descritivo }\end{array}$ & $\begin{array}{c}\text { O estudo evidencia as políticas de } \\
\text { desmonte do SUS e as } \\
\text { peculiaridades no Rio de Janeiro } \\
\text { com problemas de } \\
\text { governabilidade que potencializa } \\
\text { essa problemática }\end{array}$ \\
\hline E5 & $\begin{array}{c}\text { Cuidado à pessoa } \\
\text { suspeita de Covid-19 } \\
\text { com sinais de gravidade } \\
\text { na atenção primária à } \\
\text { saúde }\end{array}$ & $\begin{array}{c}\text { Florianó } \\
\text { polis - } \\
\text { SC, } \\
2020\end{array}$ & $\begin{array}{c}\text { Descrever o processo de } \\
\text { elaboração e a implementação de } \\
\text { um checklist de cuidados à pessoa } \\
\text { suspeita de Covid-19 com sinais } \\
\text { de gravidade na APS }\end{array}$ & $\begin{array}{l}\text { Relato de } \\
\text { experiência }\end{array}$ & $\begin{array}{l}\text { Um checklist, bem elaborado, } \\
\text { claro e objetivo, aplicado às } \\
\text { pessoas com sintomas } \\
\text { respiratórios grave, suspeitos de } \\
\text { Covid-19 configura uma } \\
\text { importante ferramenta que } \\
\text { qualifica a assistência }\end{array}$ \\
\hline E6 & $\begin{array}{l}\text { Atendimento } r \\
\text { gestantes na atenção } \\
\text { primária a saúde pela } \\
\text { enfermeira durante a } \\
\text { pandemia do SARS- } \\
\text { COV-2 }\end{array}$ & $\begin{array}{l}\text { Cratéus- } \\
\text { CE, } \\
2020\end{array}$ & $\begin{array}{l}\text { Descrever o papel do enfermeiro } \\
\text { na realização de consulta pré- } \\
\text { natal durante a pandemia no } \\
\text { âmbito da APS }\end{array}$ & $\begin{array}{l}\text { Estudo } \\
\text { descritivo do } \\
\text { tipo relato } \\
\text { de } \\
\text { experiência }\end{array}$ & $\begin{array}{l}\text { É importante aproveitar a consulta } \\
\text { pré-natal para trabalhar educação } \\
\text { em saúde com as gestantes } \\
\text { reforçando as orientações sobre a } \\
\text { Covid-19, entre outras }\end{array}$ \\
\hline E7 & $\begin{array}{l}\text { Manejo do cuidado e a } \\
\text { educação em saúde na } \\
\text { atenção primária na } \\
\text { pandemia } \\
\text { coronavírus } \\
\end{array}$ & $\begin{array}{l}\text { Monte } \\
\text { Azul - } \\
\text { MG } \\
2020\end{array}$ & $\begin{array}{llll}\text { Refletir sobre o manejo } & \text { do } \\
\text { cuidado e s educação em saúde na } \\
\text { atenção } & \text { primária } & \text { no } \\
\text { enfrentamento da pandemia } & \text { da } \\
\text { Covid-19 } & & \\
\end{array}$ & $\begin{array}{l}\text { Estudo } \\
\text { descritivo do } \\
\text { tipo relato } \\
\text { de } \\
\text { experiência }\end{array}$ & $\begin{array}{l}\text { O estudo relata a adoção de } \\
\text { medidas de enfrentamento à } \\
\text { Covid-19 que possibilitou a } \\
\text { reorganização do processo de } \\
\text { trabalho }\end{array}$ \\
\hline E8 & $\begin{array}{l}\text { Agentes comunitários } \\
\text { de saúde frente a Covid- } \\
19 \text {, vivencias juntos aos } \\
\text { profissionais da } \\
\text { enfermagem }\end{array}$ & $\begin{array}{l}\text { Icó- } \\
\text { Ceará, } \\
2020\end{array}$ & $\begin{array}{l}\text { Descrever a experiência } \\
\text { vivenciadas por enfermeiras e } \\
\text { agentes comunitários de saúde } \\
\text { para o enfrentamento da Covid- } \\
19\end{array}$ & $\begin{array}{l}\text { Relato de } \\
\text { experiência }\end{array}$ & $\begin{array}{l}\text { Observou-se a importância do } \\
\text { papel do ACS no enfrentamento à } \\
\text { pandemia da Covid-19, } \\
\text { destacando seu papel de educador } \\
\text { em saúde imprescindível nos } \\
\text { territórios e o protagonismo na } \\
\text { ESF }\end{array}$ \\
\hline E9 & $\begin{array}{l}\text { Atenção primária a } \\
\text { saúde frente a Covid- } \\
\text { 19relato de experiência } \\
\text { de um centro de saúde }\end{array}$ & $\begin{array}{l}\text { Bahia, } \\
2020\end{array}$ & $\begin{array}{l}\text { Relatar as estratégias de } \\
\text { enfrentamento de um centro de } \\
\text { saúde da atenção primária num } \\
\text { município do sul da Bahia }\end{array}$ & $\begin{array}{l}\text { Relato de } \\
\text { experiência }\end{array}$ & $\begin{array}{l}\text { Os desafios da pandemia } \\
\text { estimularam profissionais a } \\
\text { reinventar novas formas de } \\
\text { atuação resgatando a educação em } \\
\text { saúde com foco na educação } \\
\text { permanente, ressignificando } \\
\text { processos de autocuidado }\end{array}$ \\
\hline
\end{tabular}




\begin{tabular}{|c|c|c|c|c|c|}
\hline E10 & $\begin{array}{l}\text { Coordenação do } \\
\text { cuidado, vigilância e } \\
\text { monitoramento de casos } \\
\text { da Covid-19 na atenção } \\
\text { primária à saúde }\end{array}$ & $\begin{array}{l}\text { Sobral - } \\
\text { CE, } \\
2020\end{array}$ & $\begin{array}{l}\text { Descrever as ações estratégicas } \\
\text { de coordenação do cuidado, } \\
\text { monitoramento e vigilância dos } \\
\text { casos de Covid-19 na atenção } \\
\text { primária à saúde }\end{array}$ & $\begin{array}{l}\text { Relato de } \\
\text { experiência }\end{array}$ & $\begin{array}{l}\text { Destaca-se o papel estratégico da } \\
\text { APS nas ações de combate à } \\
\text { Covid-19, sobretudo na redução } \\
\text { da transmissão comunitária, no } \\
\text { monitoramento dos casos e a } \\
\text { vigilância em cada fase da } \\
\text { pandemia }\end{array}$ \\
\hline E11 & $\begin{array}{l}\text { Reflexões sobre o papel } \\
\text { da atenção primária a } \\
\text { saúde na pandemia de } \\
\text { Covid-19 }\end{array}$ & $\begin{array}{l}\text { Rio de } \\
\text { Janeiro } \\
2020\end{array}$ & $\begin{array}{l}\text { Refletir sobre o potencial de } \\
\text { atuação da APS em relação a } \\
\text { pandemia por meio de seus } \\
\text { atributos. }\end{array}$ & $\begin{array}{l}\text { Estudo } \\
\text { descritivo }\end{array}$ & $\begin{array}{l}\text { Descreve cada atributo da atenção } \\
\text { primária, salientando sua } \\
\text { importância como mecanismo de } \\
\text { enfrentamento á pandemia da } \\
\text { Covid-19 }\end{array}$ \\
\hline E12 & $\begin{array}{l}\text { Estratificação do grau } \\
\text { de vulnerabilidade à } \\
\text { doença pelo novo } \\
\text { coronavírus (Covid-19) } \\
\text { em territórios adscritos } \\
\text { da estratégia de saúde } \\
\text { da família, no município } \\
\text { de Crato, Ceará }\end{array}$ & $\begin{array}{l}\text { Crato - } \\
\text { CE } \\
2020\end{array}$ & $\begin{array}{l}\text { Descrever intervenções } \\
\text { preventivas realizadas junto às } \\
\text { famílias mais vulneráveis }\end{array}$ & $\begin{array}{l}\text { Estudo } \\
\text { descritivo } \\
\text { transversal } \\
\text { do tipo } \\
\text { relato de } \\
\text { experiência }\end{array}$ & $\begin{array}{l}\text { Este estudo embasou a elaboração } \\
\text { de intervenções preventivas de } \\
\text { caráter formativo com o intuito de } \\
\text { promover o autocuidado junto as } \\
\text { famílias, monitorando } \\
\text { comorbidade e fatores sócio } \\
\text { econômico }\end{array}$ \\
\hline E13 & $\begin{array}{l}\text { O papel da atenção } \\
\text { primária no combate ao } \\
\text { Covid-19 }\end{array}$ & $\begin{array}{l}\text { Rio de } \\
\text { Janeiro } \\
2020\end{array}$ & $\begin{array}{l}\text { Analisar o papel da atenção } \\
\text { primária á saúde na luta contra o } \\
\text { Covid-19 e seu impacto na saúde } \\
\text { pública e as futuras perspectivas }\end{array}$ & $\begin{array}{l}\text { Estudo } \\
\text { descritivo }\end{array}$ & $\begin{array}{l}\text { O melhor instrumento no controle } \\
\text { da Covid-19 é a prevenção, sendo } \\
\text { a APS o lugar ideal para } \\
\text { desenvolvimento dessas ações, no } \\
\text { entanto se faz necessário o } \\
\text { fortalecimento da APS }\end{array}$ \\
\hline
\end{tabular}

Fonte: Autores (2021).

\subsection{Caracterização da atuação da atenção primária à saúde no enfrentamento da pandemia da Covid-19 no Brasil}

$\mathrm{Na}$ análise dos artigos selecionados, identificou-se duas categorias analíticas que podem caracterizar o funcionamento da atenção primária no Brasil no contexto da pandemia da Covid-19. Os autores enfatizaram o processo de 'reorganização do processo de trabalho' que foi necessário desenvolver e as 'atividades' (mantidas, adaptadas ou criadas) que foram realizadas nesse período.

A atenção primária à saúde é descrita por oito artigos como um importante dispositivo de enfrentamento à Covid-19 (Maia, Agostini \& Azize, 2020; Hermida et al., 2020; Dias \& Ribeiro, 2020; Duarte et al., 2020; Rios et al., 2020; Ximenes Neto et al., 2020; Alves, 2020; Farias et al., 2020). Destes, seis trabalhos mencionam os atributos da APS (primeiro contato, territorialização, longitudinalidade, integralidade, coordenadora do cuidado e orientação familiar e comunitária) como mecanismo no combate à pandemia (Maia, Agostini \& Azize, 2020; Hermida et al., 2020; Dias \& Ribeiro, 2020; Duarte et al., 2020; Alves, 2020; Farias et al., 2020).

Esses estudos corroboram com documento publicado pela Associação Brasileira de Saúde Coletiva (ABRASCO) que se posicionou reiterando a importância da atuação da APS no enfrentamento à pandemia da Covid-19, através de seus atributos, especialmente aqueles que tange o aspecto territorial e comunitário (ABRASCO, 2020). A APS é reconhecida como essencial no enfrentamento do cenário pandêmico da atualidade e apresenta resultados favoráveis relacionados, principalmente, à sua forte característica de capilarização com enfoque territorial e coletivo, tendo competência para desenvolver estratégias capazes de conter a transmissão do patógeno (Belfort, Costa \& Monteiro, 2021; Giovanella et al., 2021). Entretanto, os autores enfatizam que desde o início da pandemia, a APS foi subestimada e subutilizada, e o foco do cuidado centrado no hospital.

Quanto às mudanças no processo de trabalho, os estudos ressaltaram a necessidade de reorganizar as agendas dos profissionais com a finalidade de qualificar o atendimento (Carletto \& Santos, 2020; Dias \& Ribeiro, 2020; Duarte et al., 2020; Rios et al., 2020; Farias et al., 2002) e a reorganização do fluxo do trabalho (Estrela et al., 2020; Carletto \& Santos, 2020; Maia, Agostini \& Azize, 2020; Hermida et al., 2020; Misquita et al., 2020; Dias \& Ribeiro, 2020; Duarte et al., 2020; Rios et 
al., 2020; Ximenes Neto et al., 2020; Farias et al., 2020). Considerando que, habitualmente, a APS atende a um público numeroso, o advento da Covid-19 ocasionou a necessidade de reorganizar no fluxo de trabalho nesse nível assistencial, com o agendamento de consultas por horário, suspensão dos atendimentos eletivos, manutenção exclusiva dos atendimentos essenciais e priorização das demandas espontâneas, com objetivo de evitar aglomeração nas unidades de saúde. Além do atendimento dos sintomáticos respiratório que ocorrem em salas separadas após triagem, a fim de evitar o contato com os demais usuários.

Essa reorganização do processo de trabalho na APS foi realizada em muitos municípios com o objetivo de garantir a continuidade do cuidado da população e atender os casos leves de Covid-19, colaborando assim, para evitar que os usuários sobrecarregassem desnecessariamente os serviços de maior complexidade. Para garantir o atendimento aos casos suspeitos ou confirmados de Covid-19, foi muito ressaltado a necessidade de separação do fluxo de atendimento dos sintomáticos respiratório, a fím de evitar o contágio dos demais usuários (Andres, Carlotto \& Leão, 2021).

Vale ressaltar dentre os aspectos relacionados ao processo de trabalho na atenção primária a necessidade de reorganizar o trabalho do Agente Comunitário de Saúde. Pois, o contexto da pandemia da Covid-19 afetou diretamente a rotina de trabalho desse profissional, que é o elo entre a comunidade e a unidade de saúde, sendo um profissional do território precisou adaptar-se a nova conjuntura, que o impôs um novo modo de trabalho com visitas peridomiciliares.

É preciso fortalecer a atuação do ACS com educação permanente melhores condições de trabalho pois sua atuação tem relevância estratégica e essencial nesse momento, tendo em vista a confiança e o vínculo que a comunidade estabeleceu esse profissional e as necessidades das ações de educação em saúde, ofertando informações e oportunizando o autocuidado (Duarte, 2020).

A educação em saúde tem sido fundamental para orientar a população sobre os principais cuidados no enfrentamento da pandemia e no estímulo para adesão as medidas de diminuição do contágio. Por isso mesmo, configura-se como a atividade realizada e mencionada em todos os estudos. A APS é um ambiente propício a promoção da educação em saúde, tendo em vista o vínculo com a comunidade e tem sido defendida pelo seu potencial no esclarecimento da população referente a Covid19 e no enfrentamento às notícias falsas que foram tão difundidas nos últimos anos, confundindo as pessoas e dificultando a implementação das ações eficazes (Giovanella et al., 2020).

Também foi possível identificar diversas atividades implantadas como estratégias de enfrentamento à pandemia da Covid-19 nos municípios. Dentre essas iniciativas estão: i) o atendimento online e o teleatendimento (Estrela et al., 2020; Dias \& Ribeiro, 2020; Rios et al., 2020; Ximenes Neto et al., 2020; Alves, 2020; Farias et al., 2020); ii) o acompanhamento e monitoramento dos casos suspeitos (Carletto \& Santos, 2020; Hermida et al., 2020; Dias \& Ribeiro, 2020; Duarte et al., 2020; Ximenes Neto et al., 2020); iii) a testagem dos casos suspeitos (Misquita et al., 2020; Ximenes Neto et al., 2020; Farias et al., 2020); iv) implementação de novas ferramentas de coleta de dados (Hermida et al., 2020; Ximenes Neto et al., 2020; Andrade et al., 2020); v) capacitação da equipe de saúde para atuar no enfrentamento à pandemia (Duarte et al., 2020; Rios et al., 2020; Ximenes Neto et al., 2020); e vi) inserção de práticas integrativas no cuidado ao profissional de saúde (Rios et al., 2020).

$\mathrm{O}$ atendimento online e o teleatendimento são importantes estratégias de acompanhamento dos casos suspeitos da Covid-19, por reduzir o risco de transmissão do patógeno e o contato do profissional de saúde, mantendo um contato contínuo com o usuário permitindo seu acompanhamento. Alguns autores têm mencionado a possibilidade de expandir esses atendimentos a outros usuários, entretanto, ressalvam a escassez de equipamentos e de acesso à internet nas unidades de saúde (Engstom et al., 2020; Cabral et al., 2020).

O monitoramento dos casos suspeitos é essencial para controlar os casos de Covid-19, entretanto necessita de ação conjunta entre a APS e vigilância epidemiológica. A fragmentação desses setores atenua o potencial dessas ações, o estudo de 
Teixeira et al. (2020) enfatiza a necessidade fortalecer esse vínculo. No estudo de Bezerra et al. (2021), são citados os inúmeros benefícios dessa aproximação para população.

A testagem da população é uma importante estratégia de controle da Covid-19, estabelecida por experiências nacionais e internacionais que evidenciam a eficácia dessa estratégia, possibilitando o isolamento do usuário e o controle da propagação do vírus (Soeiro et al., 2020).

No estudo de Teixeira et al. (2020), os autores explanaram sobre o problema que afetou os profissionais que atuam diretamente no enfrentamento da pandemia da Covid-19, onde aponta estratégia para proteção e assistência desses profissionais, dentre elas a capacitação do pessoal para homogeneizar o processo de trabalho da equipe enfatizando o uso de tecnologia digital, equipamentos de proteção individual (EPI), entre outros.

Outro estudo descreveu a utilização das PICS como apoio aos profissionais de saúde no campo da saúde mental (Rios et al., 2020). A pandemia gerou preocupação e apreensão generalizada na população, muitas incertezas e insegurança que atingiu a todos, especialmente os profissionais da saúde, onde a ocupação laboral os expunham mais ao risco do contágio e muitos precisaram se afastar de seus familiares, tornando esse processo ainda mais difícil. Nesse contexto, alguns municípios implantaram ações específicas de cuidado aos profissionais de saúde e o artigo de Oliveira et al. (2020) corrobora a relevância desse cuidado tendo em vista os resultados obtidos em seu estudo em práticas similares.

Em decorrência do risco de transmissão da Covid-19, alguns serviços da APS tiveram suas atividades suspensas. Entretanto, os artigos selecionados neste estudo, destacam a importância de se manter o funcionamento da rede de atenção primária. Foi ressaltado, ainda, a importância de se manter a vacinação (Carletto \& Santos, 2020; Duarte et al., 2020; Rios et al., 2020); a relevância da continuidade do atendimento às gestantes (Estrela et al., 2020; Carletto \& Santos, 2020; Misquita et al., 2020; Dias \& Ribeiro, 2020; Duarte et al., 2020; Rios et al., 2020); e a importância do acompanhamento aos grupos de risco (Carletto \& Santos, 2020; Maia, Agostini \& Azize, 2020; Misquita et al., 2020; Dias \& Ribeiro, 2020; Duarte et al., 2020; Rios et al., 2020;Ximene Neto et al, 2020; Farias et al., 2020).

O estudo de Medina et al. (2020) analisa a possibilidade da atuação da APS durante a pandemia citando a necessidade de elaborar planos de gerenciamento de riscos fortalecendo a atuação das equipes no território e incentivando a continuidade das ações da mesma, ampliando apoio aos grupos de riscos e aos vulneráveis, mantendo a realização de vacinação e demais readequação da rotina e adição das tecnologias da informação no processo do cuidado.

Nos estudos selecionados não foi mencionado a atuação de outros programas que compõe a atenção primária, como: Academia da Saúde; Consultório na Rua, Núcleo Ampliado de Saúde da Família e Atenção Básica, entre outros. A ausência de estudos sobre os demais dispositivos da atenção primária é fruto do processo de desmonte que a APS vem sofrendo no Brasil que vem reforçando o modelo reducionista de cuidado em saúde, onde os mesmos não são vistos como local de enfrentamento à pandemia e essa redução é associada a supervalorização dos serviços de alta complexidade como esse local de enfrentamento e linha de frente (Giovanella et al, 2020).

\subsection{Dificuldades enfrentadas pela atenção primária à saúde no enfrentamento da pandemia da Covid-19 no Brasil}

Uma série de mudanças permeia a atenção primária a partir do surgimento da pandemia, tendo em vista tal complexidade, os autores destacam as principais dificuldades e desafios enfrentados: a vulnerabilidade dos profissionais no enfrentamento à Covid-19 (Fernandes \& Ortega, 2020; Maia, Agostini \& Azize, 2020; Duarte et al., 2020); o desprovimento, em quantidade ou qualidade, dos Equipamentos de Proteção Individual para os profissionais (Fernandes \& Ortega, 2020; Carletto \& Santos, 2020; Maia, Agostini \& Azize, 2020; Duarte et al., 2020; Rios et al., 2020; Farias et al., 2020); a fragilidade no conhecimento sobre o uso correto de EPI (Rios et al., 2020; Ximenes Neto et al, 2020; Farias et al., 2020). 
Inicialmente, evidencia-se que a provisão de EPI para os profissionais caracterizou-se como uma das principais dificuldades advindas com a pandemia. Essa escassez se deu pelo aumento da demanda por EPI em decorrência da pandemia, somado ao pânico da população (Guimarães et al., 2020).

Ademais, outro entrave encontrado nos estudos foi a dificuldade dos usuários em acessar a rede de atenção à saúde (RAS) (Fernandes \& Ortega, 2020; Maia, Agostini \& Azize, 2020). Segundo Daumas et al. (2020) a atuação plena da APS não é alcançada por deficiência da rede de atenção à saúde, especialmente em relação à Covid-19, pois, a APS é a coordenadora da rede e a ordenadora do cuidado, necessitando do apoio da RAS para garantir a continuidade do cuidado nos casos que demandam assistência em outro nível de complexidade.

Foi elencado ainda como desafios a resistência da população na adesão às medidas de prevenção (Dias \& Ribeiro, 2020; Andrade et al., 2020) e a dificuldade no enfrentamento às falsas notícias a respeito da pandemia (Misquita et al., 2020; Dias \& Ribeiro, 2020; Duarte et al., 2020; Rios et al., 2020). Essas problemáticas prejudicam o trabalho dos profissionais, pesquisadores e gestores da saúde, uma vez que as falsas notícias costumam ir de encontro com algumas medidas de prevenção e corroboram com o descumprimento das mesmas pela população (Palacio; Takenami,2020).

Os estudos ainda elencaram como dificuldades a redução do número de profissionais (Fernandes; Ortega, 2020; Maia, Agostini \& Azize, 2020; Rios et al., 2020; Farias et al., 2020); e a dificuldade em testar e notificar os casos de Covid-19 (Fernandes; Ortega, 2020). Essa redução no quadro de profissionais na RAS, com o afastamento dos profissionais do grupo de risco, em consonância do aumento da demanda por profissionais da saúde também foi um dos principais entraves no período de pandemia. Em seu estudo, Guimarães et al. (2020) relatou a necessidade de ampliar o quadro de profissionais em seu município em decorrência desses entraves.

Por fim, foi identificado como desafio a garantia da integralidade do cuidado (Misquita et al., 2020; Dias \& Ribeiro, 2020; Duarte et al., 2020; Rios et al., 2020; Alves, 2020). A precarização da integralidade do cuidado ocorreu diante dos demais obstáculos apresentados e em decorrência da focalização da assistência nos serviços hospitalares na pandemia em detrimento das demais necessidades de saúde da população (Vitória \& Campos, 2020).

\section{Conclusão}

O presente estudo se propôs a analisar a atuação da atenção primária no enfrentamento à pandemia da Covid-19, considerando sua relevância e resolubilidade no combate às epidemias, norteada por seus atributos.

O mesmo identificou algumas evidências sobre o papel relevante da APS no enfrentamento à Covid-19, elencando as principais atividades e ações desenvolvidas. Dentre elas observou-se a necessidade de mudanças no processo de trabalho dos profissionais para atender a nova realidade advinda com a pandemia, através da mudança no fluxo de atendimento, inserção de tecnologias da informação no processo de trabalho com ênfase nas ações de educação em saúde, além das ações integradas entre a vigilância em saúde e a APS, essas modificações com a inserção dessas novas práticas, como o teleatendimento, contribuíram para o fortalecimento da atuação da atenção primária no contexto da pandemia.

Entretanto, foram identificadas também diversas fragilidades na APS como: a insipiente capacidade de articulação com a rede de atenção; fragilidades na estrutura do SUS e dos municípios como na disponibilidade de equipamentos (telefones e acesso à internet) para o monitoramento de casos e estratégias de educação em saúde; e a ausência de um delineamento de estratégias a nível nacional com relação a atuação da APS na pandemia.

Ressalta-se ainda a importância de fortalecer a APS, especialmente no atual cenário da pandemia, investindo na sua expansão e qualificação da atenção através da oferta de condições adequadas de trabalho e valorização dos seus trabalhadores. Essa é uma necessidade urgente tendo em vista diversos desafios que a APS tem enfrentado no Brasil nos últimos anos, e o seu papel estratégico na consolidação do SUS e na garantia do cuidado integral em saúde da população. 
Outro entrave importante no desenvolvimento desse trabalho foi a escassez de produção científica acerca da APS no contexto da pandemia, tanto estudos com relação a Estratégia de Saúde da Família, quanto aos outros programas da APS, como o NASF-AB, Academia da Saúde e outros.

Diante disso, se faz necessário ampliar a produção de trabalhos científicos a respeito da atuação da APS no contexto da pandemia em diferentes regiões do Brasil e avaliar a efetividade de suas ações.

\section{Referências}

Alves, M. T.G. (2020) Reflexões sobre o papel da Atenção Primária à Saúde na pandemia de COVID-19. Revista Brasileira de Medicina de Família e Comunidade, 15(42),2496. https://rbmfc.org.br/rbmfc/article/view/2496.

Amaral, L. S. do, Santos, A. L. P. dos, Figueiredo, M. P. S. de, Ferreira, D. S. de A., Silva, J. E., Santos, H. C. T. dos, Rocha, J. S., Gomes, D. A., \& Moreira, G. R. (2020). Interiorização do Covid-19: Uma análise da evolução dos casos/10 mil habitantes em municípios da Microrregião de Garanhuns no Estado de Pernambuco, através de modelos de Regressão não linear. Research, Society and Development, 9(9), e293996582. https://doi.org/10.33448/rsd-v9i9.6582.

Andrade, A. O., Fernandes, P. A. de S., Pereira, G. F., Brito, F. L. S. de, Canuto, A. F. A., Sampaio, J. M. F., Peixoto, M. Q., Luna, A. L. N. da L., Pereira, S. M. P. D., \& Brito Júnior, F. E. de. (2020). Estratificação do Grau de vulnerabilidade à doença pelo novo coronavírus (COVID-19) em territórios adscritos da Estratégia da Saúde da Família no Município de Crato, Ceará. Research, Society and Development, 9(10), e549108241. https://doi.org/10.33448/rsdv9i10.8241.

Andres, S., Carlotto, A., \& Leão, A. (2021). A organização e estruturação do serviço de saúde na APS para o enfretamento da Covid-19: relato de experiência. APS em Revista, 3(1), 09-15. https://doi.org/10.14295/aps.v3i1.137

Associação Brasileira de Saúde Coletiva. (2020). Fortalecer a Estratégia Saúde da Família no enfrentamento da Covid-19: Posicionamento da Rede APS. Rio de Janeiro:Rede APS.https://redeaps.org.br/2020/06/25/documento-tecnico-de-posicionamento-da-rede-aps-fortalecer-a-esf-no-enfrentamento-da-Covid-19/.

Barbosa, S. P. (2020) O novo coronavírus na perspectiva da atenção primária à saúde. https://www2.ufjf.br/noticias/wp-content/uploads/sites/2/2020/04/onovo-coronavirus-na-perspectiva-da-aps-simone-pinho-final1.pdf.

Belfort, I., Costa, V., \& Monteiro, S. (2021). Acolhimento na estratégia saúde da família durante a pandemia da Covid-19. APS em Revista, 3(1), 03-08. https://doi.org/10.14295/aps.v3i1.139.

Bezerra, I. N. M., Silva, A. V. da, Farias, S. J. M. de, Oliveira, M. H. M. de, Araujo, A. A. de, Santos, T. M., Silva, D. F. da, Leite, A. F. B., \& Sousa, F. de O. S. (2021). Integração ensino e serviço no contexto da pandemia de COVID-19: relato de experiência da práxis dos residentes sanitaristas na vigilância epidemiológica. Vigilância Sanitária Em Debate: Sociedade, Ciência \& Tecnologia-Visa Em Debate, 9(1), 55-60. https://doi.org/10.22239/2317-269x.01771.

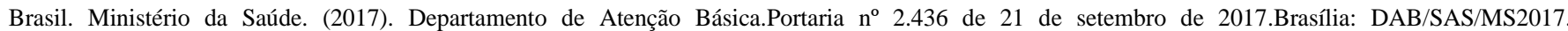
https://bvsms.saude.gov.br/bvs/saudelegis/gm/2017/prt2436_22_09_2017.html.

Brasil. Ministério da Saúde. (2020). O que é a COVID-19?.https://www.gov.br/saude/pt-br/coronavirus/o-que-e-o-coronavirus.

Cabral, E. R. de M., Bonfada, D., Melo, M. C. de, Cesar, I. D., Oliveira, R. E. M. de, Bastos, T. F., Bonfada, D., Machado, L. O., Rolim, A. C. A., \& Zago, A. C. W. (2020). Contributions and challenges of the Primary Health Care across the pandemic COVID-19. InterAmerican Journal of Medicine and Health, 3,1 12. https://doi.org/10.31005/iajmh.v3i0.87.

Campos, G.W.S. (2020). O pesadelo macabro da Covid-19 no Brasil: entre negacionismos e desvarios. Trab. educ. saúde, 18(3).http://www.scielo.br/scielo.php?script=sci_arttext\&pid=S1981-77462020000300302\&lng=en\&nrm=iso.

Carletto, A. F.\& Santos, F.F. dos. (2020). A atuação do dentista de família na pandemia do Covid-19: o cenário do Rio de Janeiro. Physis,30(3). http://www.scielo.br/scielo.php?script=sci_arttext\&pid=S0103-73312020000300309\&lng=en\&nrm=isso.

Carvalheiro, J. da R. (2020). Os coletivos da Covid-19. Estudos Avançados, 34(99),7-23. https://doi.org/10.1590/s0103-4014.2020.3499.002.

Costa, A. M., Rizzotto, M. L. F.\& Lobato, L.V. C. (2020). Na pandemia da Covid-19, o Brasil enxerga o SUS. Saúde debate, Rio de Janeiro,44(125),289296.http://www.scielo.br/scielo.php?script=sci_arttext\&pid=S0103-11042020000200289\&lng=en\&nrm=iso.

Daumas, R.P., Silva, G. A.,Tasca, R.,Brasil,Leite, I.C., P.,Greco, D. B.,Grabois, V. \& Campos, G.W.S.(2020) O papel da atenção primária na rede de atenção à saúde no Brasil: limites e possibilidades no enfrentamento da COVID-19. Cad. Saúde Pública,36(6). http://www.scielo.br/scielo.php?script=sci_arttext\&pid=S0102-311X2020000600503\&lng=en\&nrm=iso.

Dias, E.G. \& Ribeiro, D. R. S. V. (2020). Manejo do cuidado e a educação em saúde na atenção básica na pandemia do Coronavírus. J. nurs. Health, 10(4)e20104020.https://periodicos.ufpel.edu.br/ojs2/index.php/enfermagem/article/view/19092/11688.

Duarte, R. B., Medeiros, L. M. F., Araújo, M. J. A. L., Cavalcante, A. S. P.,Souza, E. C., Alencar, O. M., Marinho, M. N. A. S. B. \& Silva, M.R.F.(2020).Agentes Comunitários de Saúde frente à COVID-19: Vivências junto aos profissionais de enfermagem. Enfermagem em Foco,11(1),252-256. http://revista.cofen.gov.br/index.php/enfermagem/article/view/3597/837.

Engstrom, E., Melo, E., Giovanella, L.,Mendes, A., Grabois,V. \& Mendonça, M. H. M. (2020).Recomendações para a organização da atenção primária à saúde no SUS no enfrentamento da COVID-19.https://portal.fiocruz.br/documento/recomendacoes-para-organizacao-da-aps-no-sus-no-enfrentamento-da-Covid-19. 
Estrela, F.M.,Silva, K. K. A.,Cruz, M. A. G. \& Pereira, N.(2020).Gestantes no contexto da pandemia da Covid-19: reflexões e desafios. Physis: Rev Saúde Coletiva. 2020; 30 (2),1-5. http://www.scielo.br/scielo.php?script=sci_arttext\&pid=S0103-73312020000200314\&lng=en\&nrm=isso.

Farias, L. A. B. G., Pessoa,M.C., Barreto, F. K.A.\& Cavalcanti, L.P.G. (2020). O papel da atenção primária no combate ao Covid-19: impacto na saúde pública e perspectivas futuras. Revista Brasileira De Medicina De Família E Comunidade, 15(42), 2455. https://doi.org/10.5712/rbmfc15(42)2455.

Fernandes, L. \& Ortega, F. (2020). A Atenção Primária no Rio de Janeiro em tempos de Covid-19. Physis, 30(3). http://www.scielo.br/scielo.php?script=sci_arttext\&pid=S0103-73312020000300308\&lng=en\&nrm=iso.

Giovanella, L., Franco, C.M., Almeida, P.F. (2020). Política Nacional de Atenção Básica: para onde vamos? Ciência \& Saúde Coletiva, 25(4):1475-1482. https://doi.org/10.1590/1413-81232020254.01842020.

Giovanella, L. Vega, R., Tejerina-Silva, H., Acosta-Ramirez, N., Parada-Lezcano, M., Ríos, G., Iturrieta, D.,Almeida, P. F. \& Feo, O. (2021). ¿Es la atención primaria de salud integral parte de la respuesta a la pandemia de Covid-19 en Latinoamérica? Trab. educ. saúde,19,1-28. http://www.scielo.br/scielo.php?script=sci_arttext\&pid=S1981-77462021000100402\&lng=en\&nrm=iso.

Giovanella, L. Martufi, V.,Mendoza, D.C.R.,Mendonça, M.H.M.,Bousquat, A.E.M.,Pereira, R.A.G. \& Medina, M.G.(2020).A contribuição da atenção primária à saúde na rede SUS de enfrentamento à Covid-19. Saúde em Debate,126(44),1-21. http://www.saudeemdebate.org.br/.

Guimarães, F., Carvalho, T., Bernardes, R., \& Pinto, J. (2020). A organização da atenção Primária à Saúde de Belo Horizonte no enfrentamento da Pandemia Covid 19: relato de experiência. APS em Revista, 2(2), 74-82. https://doi.org/10.14295/aps.v2i2.128.

Hermida, P. M. V., Silveira, N. D., Bringhenti, L. J., Bugs, T. S., Miotto, P., Chiari, M. F. \& Sulis, P. M. (2020).Cuidados à pessoa suspeita de COVID-19 com sinais de gravidade na Atenção Primária à Saúde. Enfermagem em Foco,11(2). http://revista.cofen.gov.br/index.php/enfermagem/article/view/4223/1005.

Lavras, C. (2011). Atenção primária à saúde e a organização de redes regionais de atenção à saúde no Brasil. Saude soc., 20(4),867874.http://www.scielo.br/scielo.php?script=sci_arttext\&pid=S0104-12902011000400005\&lng=en\&nrm=iso.

Maia, A. C., Agostini, R. \& Azize, R. L. (2020).Sobre jogos de (des)montar: Covid-19 e Atenção Primária à Saúde. Physis, 30(3). http://www.scielo.br/scielo.php?script=sci_arttext\&pid=S0103-73312020000300314\&lng=en\&nrm=isso.

Medina, M. G., Giovanella, L., Bousquat, A.E.M.,Mendonça,M.H.M., Aquino,R.(2020). Atenção primária à saúde em tempos de COVID-19: o que fazer?. Cad. Saúde Pública,36(8).http://www.scielo.br/scielo.php?script=sci_arttext\&pid=S0102-311X2020000800502\&lng=en\&nrm=iso.

Melo, E. A., Mendonça, M. H. M, Oliveira, J. R., Andrade, G. C. L (2018). Mudanças na Política Nacional de Atenção Básica: entre retrocessos e desafios. Saúde em Debate, 42,38-51. https://doi.org/10.1590/0103-11042018S103.

Morosini, M. V. G. C., Fonseca, A. F., Baptista, T. W. F. (2020). Previne Brasil, Agência de Desenvolvimento da Atenção Primária e Carteira de Serviços: radicalização da política de privatização da atenção básica?. Cad. Saúde Pública, 36 (9). https://doi.org/10.1590/0102-311X00040220.

Noronha, K. V. M. S. Guedes, G. R.,Turra, C. M., Andrade, M. V., Botega, L., Nogueira, D., Calazans, J. A., Carvalho, L., Servo, L., Ferreira, M. F. (2020). Pandemia por COVID-19 no Brasil: análise da demanda e da oferta de leitos hospitalares e equipamentos de ventilação assistida segundo diferentes cenários. Cad. Saúde Pública,36(6). http://www.scielo.br/scielo.php?script=sci_arttext\&pid=S0102-311X2020000605004\&lng=en\&nrm=iso.

Oliveira, M. A., Monteiro, L., Oliveira, R., Moreira, T., Marques, A. C., Silva, U., Oliveira, N., Pereira, G., Silva, A. C., \& Santana, R. (2020). A prática do núcleo de apoio à saúde da família do Recife no enfrentamento à pandemia COVID-19. APS em Revista,2(2),142150.https://apsemrevista.org/aps/article/view/96.

Paim,J.S.(2018).Sistema Único de Saúde (SUS) aos 30 anos. Ciênc. saúde coletiva,23(6), 1723-1728. http://www.scielo.br/scielo.php?script=sci_arttext\&pid=S1413-81232018000601723\&lng=en\&nrm=iso.

Palácio, M. A. V., \& Takenami, I. (2020). Em tempos de pandemia pela COVID-19: o desafio para a educação em saúde. Vigilância Sanitária Em Debate: Sociedade, Ciência \& Tecnologia - Visa Em Debate, 8(2), 10-15. https://doi.org/10.22239/2317-269x.01530.

Pinto, L.F.; Giovanella, L. (2018). Do Programa à Estratégia Saúde da Família: expansão do acesso e redução das internações por condições sensíveis à atenção básica (ICSAB). Ciência \& Saúde Coletiva, 23(6), 903-1914. https://www.scielo.br/scielo.php?script=sci_arttext\&pid=S1413$81232018000601903 \& \operatorname{lng}=$ pt\&tlng=pt.

Rios, A. F. M. Lira, L. S. S. P.,Reis, I. M. \& Silva, G. A.(2020).Atenção Primária à Saúde frente à COVID-19: Relato de experiência de um Centro de Saúde. Enfermagem em Foco,11(1). http://revista.cofen.gov.br/index.php/enfermagem/article/view/3666/836.

Santos Júnior, C. J. dos, \& Rocha, T. J. M. (2020). Dois milhões de casos da COVID-19 no Brasil. Medicina (Ribeirão Preto), 53(2), 201-203. https://doi.org/10.11606/issn.2176-7262.v53i2p201-203.

Sarti, T. D., Lazarini, W. S., Fontenelle, L. F., \& Almeida,A. P. S. C. (2020).Qual o papel da Atenção Primária à Saúde diante da pandemia provocada pela COVID-19?. Epidemiol.Serv.Saúde, 29(2).http://www.scielo.br/scielo.php?script=sci_arttext\&pid=S2237-96222020000200903\&lng=en\&nrm=iso.

Misquita, M. S., Silva, P. G., Braz, G. A., Sousa, A. B. A. G., Melo, D. F.C., \& Melo, F. N. P. (2020). Atendimento de gestantes na atenção primária a saúde pela enfermagem durante a pandemia do SARS-COV-2.Nursing (São Paulo), 23(269), 4723-4730. https://doi.org/10.36489/nursing.2020v23i269p4723-4730.

Soeiro, R. E., Bedrikow, R., Ramalho, B. D. de S., Niederauer, A. J. S., Souza, C. V. de, Previato, C. S., Martins, D. B., Dias, T. M., Ribas Freitas, A. R., \& Dimarzio, G. (2020). Atenção Primária à Saúde e a pandemia de COVID-19: reflexão para a prática. InterAmerican Journal of Medicine and Health,3.https://doi.org/10.31005/iajmh.v3i0.83.

Souza, D. O. (2020). O subfinanciamento do Sistema Único de Saúde e seus rebatimentos no enfrentamento da Covid19. Physis,30(3).http://www.scielo.br/scielo.php?script=sci_arttext\&pid=S0103-73312020000300312\&lng=en\&nrm=iso. 
Research, Society and Development, v. 10, n. 8, e42010817359, 2021

(CC BY 4.0) | ISSN 2525-3409 | DOI: http://dx.doi.org/10.33448/rsd-v10i8.17359

Souza, M. T., Silva, M. D., \& Carvalho, R. (2010). Revisão integrativa: o que é e como fazer. Einstein (São Paulo), 8(1), 102-106. https://doi.org/10.1590/s1679-45082010rw1134

Teixeira, C. F. S, Soares, C. M., Souza, E. A, Lisboa, E. S, Pinto, I. C. M., Andrade, L. \&Esperidião,M. A. (2020).A saúde dos profissionais de saúde no enfrentamento da pandemia de Covid-19. Ciênc. saúde coletiva, 25(9), 3465-3474. http://www.scielo.br/scielo.php?script=sci_arttext\&pid=S1413$81232020000903465 \& \operatorname{lng}=$ en $\&$ nrm $=$ iso.

They, N. H. (2020). Uma breve linha do tempo.https://www.ufrgs.br/coronaviruslitoral/uma-breve-linha-do-tempo/.

They, N.H.(2020) COVID-19: Evidências científicas da eficácia do distanciamento social. https://www.ufrgs.br/coronaviruslitoral/Covid-19-evidenciascientificas-da-eficacia-do-distanciamento-social/.

Vitória, A. M.; Campos, G. W. S. (2020).Dica do gestor: Só com APS forte o sistema pode ser capaz de achatar a curva de crescimento da pandemia e garantir suficiência de leitos UTI. http://www.cosemssp.org.br/noticias/dicadogestor-so-com-aps-forte-o-sistema-pode-ser-capaz-de-achatar-a-curva-de-crescimentoda-pandemia-e-garantir-suficiencia-de-leitos-uti/.

Ximenes Neto, F. R. G., Araújo, C.R.C., Silva, R.C.C., Ribeiro,M.A.,Sousa,L.A.,Serafim, T.F., Dorneles, J.A. \& Gadelha, L.A. (2020).Coordenação do cuidado, vigilância e monitoramento de casos da COVID-19 na Atenção Primária à Saúde. Enfermagem em Foco,11(1).http://revista.cofen.gov.br/index.php/enfermagem/article/view/3682. 